\title{
Photoionisation of the tropyl radical
}

\author{
Kathrin H. Fischer ${ }^{1}$, Patrick Hemberger ${ }^{*}$, Andras Bodi ${ }^{2}$ and Ingo Fischer ${ }^{* 1}$
}

\section{Full Research Paper}

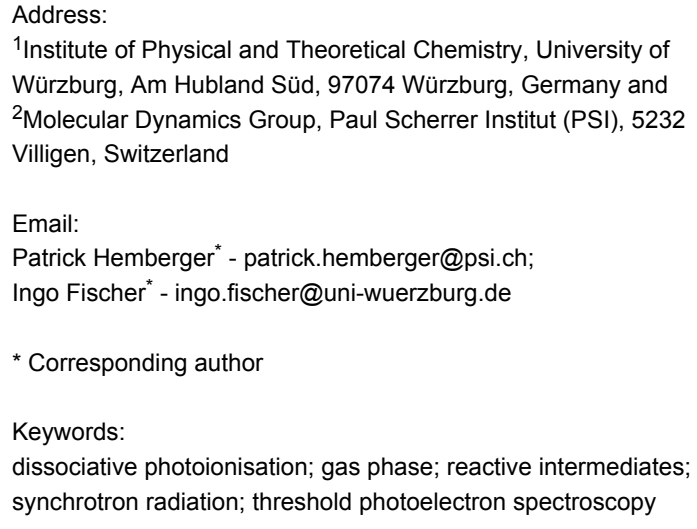

Beilstein J. Org. Chem. 2013, 9, 681-688. doi:10.3762/bjoc. 9.77

Received: 23 January 2013

Accepted: 13 March 2013

Published: 09 April 2013

This article is part of the Thematic Series "New reactive intermediates in organic chemistry".

Guest Editor: G. Bucher

(C) 2013 Fischer et al; licensee Beilstein-Institut. License and terms: see end of document.

\begin{abstract} tion of tropyl is observed, leading to the formation of $\mathrm{C}_{5} \mathrm{H}_{5}{ }^{+}$and $\mathrm{C}_{2} \mathrm{H}_{2}$.

\section{Introduction}

Organic radicals are known to be ubiquitous reactive intermediates in chemistry, biology and material science [1]. Studies on isolated radicals conducted in our group [2] yield their intrinsic properties, which are essential for understanding the reactivity of radicals in both the gas and condensed phase. Here we present a detailed study on the photoionisation of the cycloheptatrienyl radical $\left(\mathrm{C}_{7} \mathrm{H}_{7}\right)$, commonly called tropyl, using synchrotron radiation.
\end{abstract}

We present a study on the photoionisation of the cycloheptatrienyl (tropyl) radical, $\mathrm{C}_{7} \mathrm{H}_{7}$, using tunable vacuum ultraviolet synchrotron radiation. Tropyl is generated by flash pyrolysis from bitropyl. Ions and electrons are detected in coincidence, permitting us to record mass-selected photoelectron spectra. The threshold photoelectron spectrum of tropyl, corresponding to the $X^{+1} \mathrm{~A}_{1}{ }^{\prime} \leftarrow X^{2} \mathrm{E}_{2}$ " transition, reveals an ionisation energy of $6.23 \pm 0.02 \mathrm{eV}$, in good agreement with Rydberg extrapolations, but slightly lower than the value derived from earlier photoelectron spectra. Several vibrations can be resolved and are reassigned to the $\mathrm{C}-\mathrm{C}$ stretch mode $v_{16}{ }^{+}$and to a combination of $v_{16}{ }^{+}$with the ring breathing mode $v_{2}{ }^{+}$. Above $10.55 \mathrm{eV}$ dissociative photoionisa-

The tropyl radical 1 and its cation $\mathbf{2}$ are depicted in Figure 1. They have been at the focus of research since the 1960s [3,4] due to their symmetry properties. The interest originated in the expected stability of the tropyl cation, which is an aromatic molecular ion according to the Hückel rules. The aromaticity was confirmed and the symmetry of the $\mathrm{C}_{7} \mathrm{H}_{7}{ }^{+}$established as $D_{7 h}$ [5]. The vibrational structure of the cation was examined by IR and Raman spectroscopy [6-8]. There are 36 normal modes with 20 distinct frequencies, owing to degeneracy. Of these twenty vibrations, four are IR- and seven Raman-active [9].

In contrast to the cation, the odd-electron neutral tropyl radical is expected to be Jahn-Teller (JT) distorted. The nature of this distortion and whether the equilibrium structure of tropyl corresponds to a distorted $C_{2 v}$ or to $D_{7 h}$ symmetry has been 


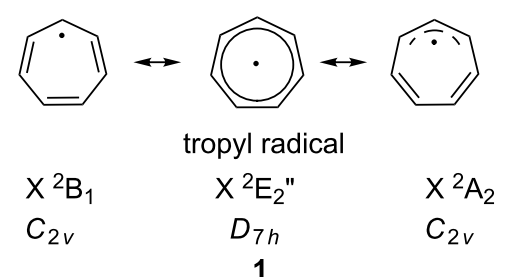

1

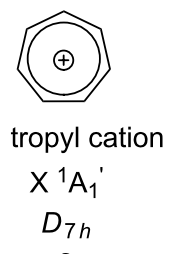

2

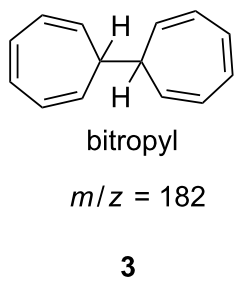

3

Figure 1: Structure of the tropyl radical 1, its cation $\mathbf{2}$ and the precursor bitropyl 3.

studied experimentally and theoretically. An early electron spin resonance (ESR) experiment found seven equivalent hydrogen atoms and a uniform spin distribution and, therefore, concluded a dynamic $D_{7 h}$ structure of the radical [10,11]. Calculations in the 1990 s reported $C_{2 v}$ symmetry due to Jahn-Teller distortion $[9,12]$. The most extensive investigation on the vibronic structure of the tropyl radical including the Jahn-Teller distortion was carried out by Miller and co-workers $[13,14]$. They found that the ${ }^{2} \mathrm{E}_{2}$ " ground state splits into two components of $C_{2 v}$ symmetry, an allylic ${ }^{2} \mathrm{~B}_{1}$ and a dienylic ${ }^{2} \mathrm{~A}_{2}$ one (Figure 1). They are stabilized by roughly $1000 \mathrm{~cm}^{-1}$ with respect to the undistorted $D_{7 h}$ saddle point [14], which corresponds to a conical intersection on the potential-energy surface. In addition to providing chemical insight, the other benefit of identifying the two $C_{2 v}$ resonance geometries on the minimum path is that it makes geometry optimisations possible by the symmetry constraint. The IR spectrum of the radical was measured in the gas phase and compared to calculations as well as to that of the benzyl radical [15].

The geometry change upon ionisation and the character of the molecular orbitals triggered interest in the photoelectron spectroscopy of tropyl. The adiabatic ionisation energy of the radical was established by Thrush and Zwolenik (6.24 eV) [3], Elder and Parr (6.236 eV, derived from a photoion yield curve) [4] and Koenig and Chang (6.28 eV) [16]. The latter used helium(I) photoelectron spectroscopy and employed bitropyl $\mathbf{3}$ as a precursor (Figure 1). This molecule proved to be an efficient source for tropyl radicals generated by pyrolysis. Furthermore, the ground and excited states of the ion have been investigated computationally $[9,17]$. In the present study we extend the previous work using imaging photoelectron-photoion coincidence (iPEPICO) techniques in combination with VUV synchrotron radiation [18-20]. Coincidence spectroscopy correlates the electron signal with the mass signal and thus permits recording of mass-selected photoelectron spectra. This is particularly advantageous in experiments on reactive intermediates where a clean sample generation cannot always be ensured. An improved resolution is obtained from analysing only the threshold electrons [18,21], i.e., electrons recorded with almost zero initial kinetic energy upon tuning the photon energy. Thus,
IR and Raman inactive ionic vibrations can often be observed and assigned in the photoelectron spectrum.

We have shown in the past that these techniques are well suited to study the photoionisation of open-shell species. Ionisation energies have been determined from vibrationally resolved photoelectron spectra for several open-shell species ranging from allyl [22] and propargyl [23] to indenyl $\left(\mathrm{C}_{9} \mathrm{H}_{7}\right)$ [24], cyclopropenylidene [25] and fulvenallenyl [26]. In the case of allyl [27,28] and propargyl [29] they are in excellent agreement with high-resolution laser studies. Such data are important for the derivation of bond dissociation energies and heats of formation of radicals, but also aid in the in situ detection of radicals in flames by photoionisation [30]. The goal of the present experimental study was to elucidate the vibrational structure of the tropyl ion ground state with threshold photoelectron spectroscopic (TPES) techniques.

\section{Results and Discussion}

The performance of the pyrolysis source can be illustrated by mass spectra at different photon energies with pyrolysis on or off, depicted in Figure 2.

As shown in the top trace of Figure 2 almost no signal is present without pyrolysis at a photon energy of $7.8 \mathrm{eV}$. At a photon energy of around $8 \mathrm{eV}$ we start to see a signal of the bitropyl precursor 3. Already at around the same energy a signal at $m / z=91$ appears. Since there is no pyrolysis, the signal has to originate from dissociative photoionisation of bitropyl. Hence, in the mass spectrum recorded at $8.7 \mathrm{eV}$ (centre trace of Figure 2) the observed masses are $m / z=91,92$ and 182, which correspond to a tropyl fragment, its ${ }^{13} \mathrm{C}$ isotopologue and the bitropyl precursor. Above a photon energy of $8.9 \mathrm{eV}$, the $m / z=104$ and 167 peaks appear in addition. They are products from higher energy dissociative photoionisation channels and are probably formed through the loss of benzene or a methyl group from the precursor, respectively. When we decrease the photon energy to $7.8 \mathrm{eV}$ again and switch on the pyrolysis, (bottom trace in Figure 2) an intense photoionisation signal is observed at the masses $m / z=91$ and 92 , which is due to the direct photoionisation of tropyl and its ${ }^{13} \mathrm{C}$ isotopologue. We 


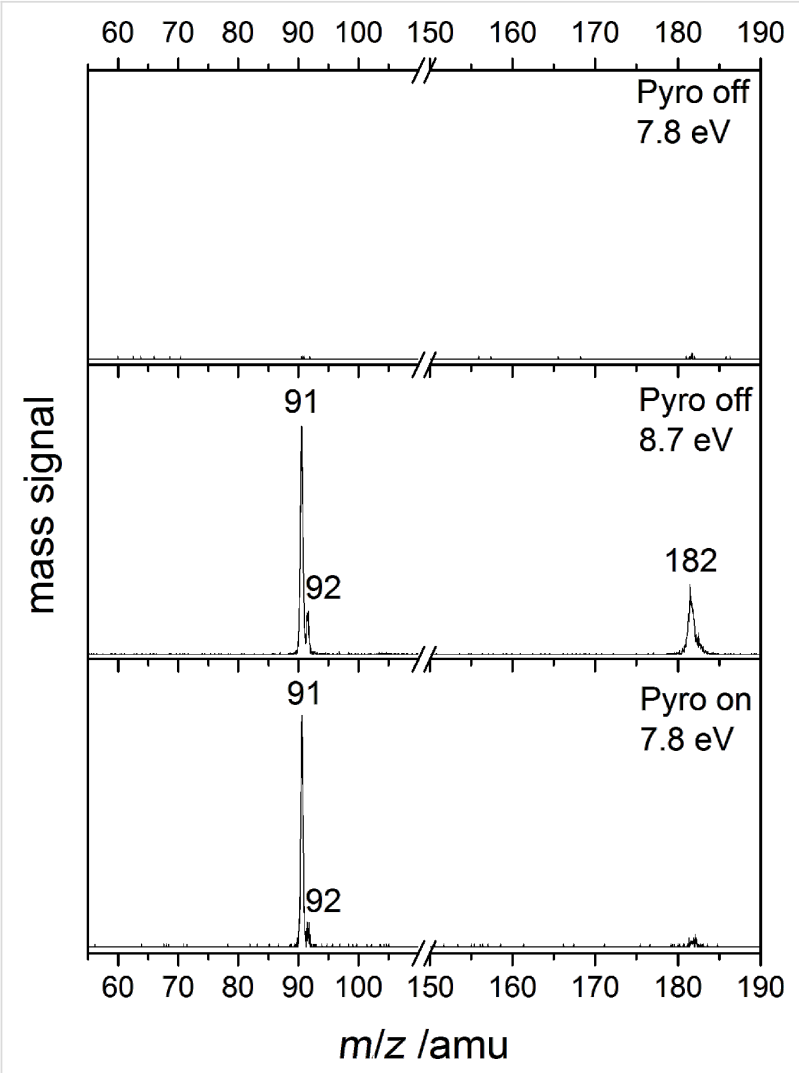

Figure 2: Mass spectra of bitropyl without pyrolysis at 7.8 and $8.7 \mathrm{eV}$ (top and centre trace) and with pyrolysis, recorded at $7.8 \mathrm{eV}$ (bottom trace).

note that the small signal at $\mathrm{m} / z=182$ never disappears completely. This is probably a result of sample contamination by an isomer of bitropyl. Thus, dissociative photoionisation may contribute to the tropyl signal at photon energies above $8 \mathrm{eV}$. Above $10.5 \mathrm{eV}$, an $m / z=65$ peak appears exclusively in the "pyrolysis on" spectra corresponding to the dissociative photoionisation of the tropyl radical, yielding the cyclopentadienyl cation and acetylene.

Figure 3 shows the region of the ionisation onset of the tropyl radical in high resolution while Figure 4 (see below) exhibits the complete spectrum with the higher energy region in lower resolution. In both mass-selected threshold photoelectron (TPE) spectra the pyrolysis was turned on. The experimental spectrum (Figure 3) shows a sharp onset with a pronounced first maximum at $6.23 \mathrm{eV}$. It is assigned to the $\widetilde{X}^{+1} \mathrm{~A}_{1}{ }^{\prime}\left(\mathrm{v}^{+}=0\right) \leftarrow$ $\widetilde{X}^{2} \mathrm{E}_{2} "(\mathrm{v} "=0)$ transition and corresponds to the adiabatic ionisation energy of the molecule. As the radical vibrational temperature is typically around $500 \mathrm{~K}$ in a continuous beam experiment [31] a contribution from hot and sequence bands cannot be excluded and could be responsible for the signal between $6.1 \mathrm{eV}$ and $6.2 \mathrm{eV}$. The small peak at around $6.12 \mathrm{eV}$ may correspond to a bending-mode hot band. Our IE value of
$6.23 \pm 0.02 \mathrm{eV}$ is in excellent agreement with the values obtained from an extrapolation of Rydberg states: a [2+1] multiphoton ionisation (MPI) study [32] reported an IE of $50177 \pm 46 \mathrm{~cm}^{-1}(6.221 \mathrm{eV})$ and the absorption experiment by Thrush and Zwolenik found a value of $6.24 \mathrm{eV}$. On the other hand, our value is slightly lower than the IE of $6.28 \mathrm{eV}$ reported by conventional photoelectron spectroscopy [16]. It is interesting to note that the ionisation energy of benzyl, the second $\mathrm{C}_{7} \mathrm{H}_{7}$ isomer, lies at $7.249 \mathrm{eV}$ [33] and is thus almost $1 \mathrm{eV}$ higher.

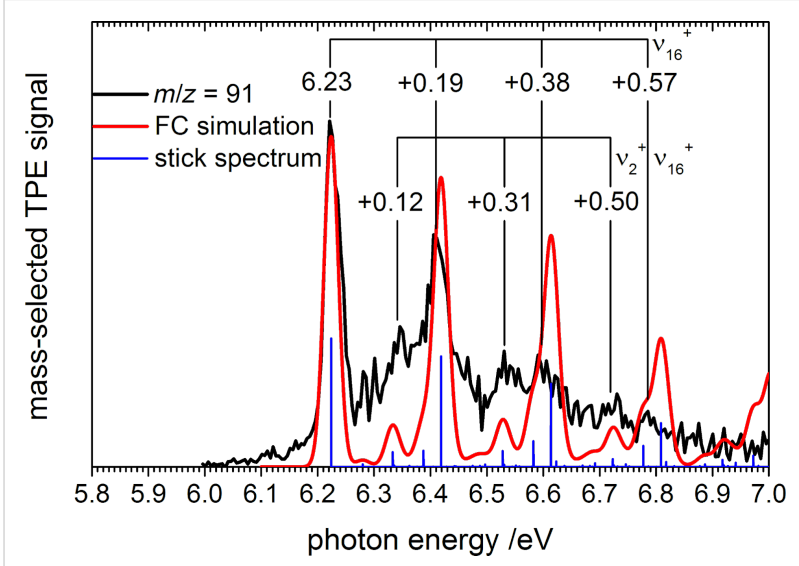

Figure 3: Threshold photoelectron spectrum of tropyl (black line). The Franck-Condon simulation (red line) is based on the computed stick spectrum (blue sticks) convoluted with $30 \mathrm{meV}$ FWHM Gaussians.

A number of peaks are apparent that belong to vibrational progressions in the cation. The first progression has a spacing of around $1530 \mathrm{~cm}^{-1}(0.19 \mathrm{eV})$. This progression has been observed before. A vibrational spacing of $1528 \pm 13 \mathrm{~cm}^{-1}$ was reported for high-lying Rydberg states and assigned to the overtone of a $\mathrm{C}-\mathrm{C}-\mathrm{C}$ bending mode of $e_{3}$ ' symmetry with a vibrational frequency of $768 \mathrm{~cm}^{-1}$ [32]. A vibrational progression with a spacing of $1424 \pm 100 \mathrm{~cm}^{-1}$ was also found in conventional PES and attributed to a $\mathrm{C}-\mathrm{C}-\mathrm{C}$ stretching mode of $e_{1}$ symmetry with a computed wavenumber of $1470 \mathrm{~cm}^{-1}$ [16]. A second pronounced peak is identified $+0.12 \mathrm{eV}$ above the origin $\left(970 \mathrm{~cm}^{-1}\right)$. This much weaker progression also appears in combination with members of the $+0.19 \mathrm{eV}$ progression, and has also been observed in the previous Rydberg state study [32]. Since it has now been firmly established that the minimum energy geometries are of $C_{2 v}$ symmetry [13,14], these earlier assignments have to be reconsidered. Only totally symmetric modes, i.e., $a_{1}$ ' modes in the case of tropyl, appear as fundamentals in a photoelectron spectrum in the absence of vibronic coupling. However, upon photoionisation the symmetry of tropyl changes from $C_{2 v}$ to $D_{7 h}$, so transitions have to be discussed in the common subgroup $C_{2 v}$. The irreducible representations $e_{1}$ ', $e_{2}$ ' and $e_{3}$ ' resolve into $\mathrm{a}_{1} \oplus \mathrm{b}_{2}$ upon going 
from $D_{7 h}$ to $C_{2 v}$. As this sum contains the totally symmetric representation $\mathrm{a}_{1}$, these doubly degenerate modes that are symmetric towards $\sigma_{\mathrm{h}}$ are expected to be symmetry allowed in a $D_{7 h} \leftarrow C_{2 v}$ transition.

In order to assign the vibrational transitions we performed a Franck-Condon (FC) simulation (Figure 3) with the FCfit program, version 2.8.8 [34] using the $\widetilde{X}^{+2} \mathrm{~B}_{1}$ allylic resonance structure of the neutral radical. We employed the geometry, frequencies and force constants of the CASSCF calculation from Stakhursky et al. [13] in the simulation. Note that their work accurately describes the Jahn-Teller distortion and thus represents the best available description of the radical potentialenergy surface. They chose a $(7,7)$ active space and employed a 6-31G(d) basis set. For the cation the input parameters were calculated by density functional theory (DFT) with the Gaussian 09 suite of programs [35], employing the B3LYP functional and a 6-31G(d) basis set. For a closed-shell molecule without vibronic distortions, a DFT approach provides the same accuracy for the geometries and frequencies as CASSCF. Our geometry and frequencies agree very well with the one reported in the literature [17]. For example a $\mathrm{C}-\mathrm{C}$ bond length $\mathrm{r}(\mathrm{C}-\mathrm{C})=1.399 \AA$ was found as compared to $\mathrm{r}(\mathrm{C}-\mathrm{C})=1.396 \AA$ by Pino et al. [17]. For all vibrational frequencies unscaled values are given below. We note that the computations have been carried out in the Abelian point groups $C_{S}$ or $C_{2 v}$. The vibrational modes were assigned following the nomenclature of Lee and Wright [9], which has also been used by Pino et al. [17].

In order to compare the FC simulation with the experiment, the stick spectrum was convoluted with a Gaussian with a FWHM (full width at half maximum) of $0.030 \mathrm{eV}$. As seen in Figure 3, the simulation is in good agreement with the experimental spectrum. The main progression with a spacing of $1530 \mathrm{~cm}^{-1}$ $(+0.19 \mathrm{eV})$ can be assigned to the doubly degenerate mode calculated at $1571 \mathrm{~cm}^{-1}\left(e_{3}, v_{16}{ }^{+}\right)$, which is an in plane $\mathrm{C}-\mathrm{C}$ stretching vibration. Upon ionisation, the $D_{7 h}$ saddle point turns into a true minimum in the absence of Jahn-Teller distortion. As this has to be associated with an adaption of the $\mathrm{C}-\mathrm{C}$ bond lengths, the corresponding vibrations are expected to be active. In the experimental spectrum and the FC simulation we also observe its first $\left(+0.38 \mathrm{eV}, 16_{0}^{2}\right)$ and second $\left(+0.57 \mathrm{eV}, 16_{0}^{3}\right)$ overtone. However, the simulation overestimates the intensity of the overtones. This is not surprising, considering that the neutral ground state geometry is delocalized and, in a crude approximation, we only use one resonance geometry and its harmonic oscillator functions. Also the second-order Jahn-Teller effect was neglected in the ground-state calculations. Still, no significant changes were observed in the simulated peak intensities when using the other $C_{2 v}$ resonance geometry of the neutral, i.e., the dienylic ${ }^{2} \mathrm{~A}_{2}$ component, even though the geometries are somewhat different. The ground state can be described as a superposition of the ${ }^{2} \mathrm{~A}_{2}$ and ${ }^{2} \mathrm{~B}_{1}$ states, and it is reassuring that they both lead to very similar FC simulations. This also explains why the harmonic-oscillator approach works reasonably well in this case.

The first member of the second progression lies $+0.12 \mathrm{eV}$ above the origin and can be assigned to the $v_{2}{ }^{+}$fundamental of $a_{1}$, symmetry in the reference geometry, $2_{0}^{1}$. A wavenumber of $v_{2}{ }^{+}$ $881 \mathrm{~cm}^{-1}$ was calculated for this ring breathing mode. This $\mathrm{v}_{2}^{+}$ mode has also been observed at $+862 \mathrm{~cm}^{-1}(+0.11 \mathrm{eV})$ in the previous Rydberg state MPI study [32]. The band is not very pronounced and the maximum is difficult to identify in our spectrum. This probably explains the deviation to the computed value. Two further peaks are assigned to be combination bands with $v_{16}{ }^{+}$, namely $2{ }_{0}^{1} 16_{0}^{1}(+0.31 \mathrm{eV})$ and $2{ }_{0}^{1} 16_{0}^{2}(+0.50 \mathrm{eV})$. An additional mode reported in the MPI spectrum at $+1284 \mathrm{~cm}^{-1}$ is also predicted by the FC-simulations as visible in the stick spectrum in Figure 3. It is buried in the red edge of the $+0.19 \mathrm{eV}$ band. We assign it to the $v_{17}{ }^{+} \mathrm{C}-\mathrm{H}$ in-plane bend of $\mathrm{e}_{3}$, symmetry, computed at $1320 \mathrm{~cm}^{-1}$.

Figure 4 presents the complete TPES up to $13.0 \mathrm{eV}$. Note that the photon energy step size changed at $7 \mathrm{eV}$. A small peak is observed at $7.25 \mathrm{eV}$. Most likely it corresponds to the adiabatic ionisation energy of the benzyl radical [36], originating either from precursor impurities or from an isomerisation in the pyrolysis. Benzyl is by about $70 \mathrm{~kJ} \mathrm{~mol}^{-1}$ more stable than tropyl $[12,37,38]$, but a high activation barrier can be assumed for the isomerisation reaction in the pyrolysis source. Although we cannot exclude that the signal in the $m / z=91$ mass channel might have some contributions from benzyl in the higher photon energy region, the benzyl signal is small compared to the tropyl one in the threshold region. Therefore the amount of the possible benzyl contamination is negligible.

With active pyrolysis an additional $\mathrm{m} / \mathrm{z}=65$ peak appears at around $8.55 \mathrm{eV}$ in the mass spectrum, which can be attributed to $\mathrm{C}_{5} \mathrm{H}_{5}{ }^{+}$. The mass-selected TPE-signal of $\mathrm{m} / \mathrm{z}=65$ is depicted as a dashed line in Figure 4. Below $10.55 \mathrm{eV}$ the signal is small and has a symmetric peak shape in the mass spectrum, as visible in the upper trace of Figure 5. Due to the small count number it is difficult to determine an accurate onset, but the signal appears around $8.55 \mathrm{eV}$. The adiabatic IE of the cyclopentadienyl radical was determined to be $8.428 \mathrm{eV}$ by high-resolution photoelectron spectroscopy [39]. Thus, the appearance of $\mathrm{C}_{5} \mathrm{H}_{5}{ }^{+}$in this energy range can be interpreted as the direct ionisation of cyclopentadienyl radical produced as a side product in the pyrolysis. Above $10.55 \mathrm{eV}$, on the other hand, the intensity rises significantly and the time-of-flight peak shape 


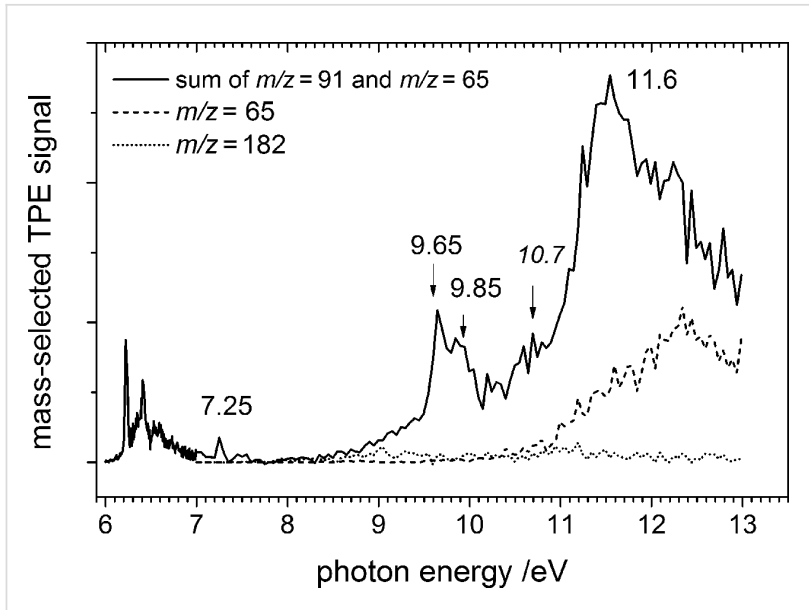

Figure 4: TPE spectrum of tropyl (solid line) and cyclopentadienyl $(\mathrm{m} / \mathrm{z}=65$, dashed line) in the 7-13 eV photon energy range. Since $\mathrm{C}_{5} \mathrm{H}_{5}{ }^{+}$is generated by dissociative photoionisation of tropyl at higher energies, the tropyl photoelectron spectrum is displayed as the sum of the two mass channels. The residual signal at the mass of the precursor is given as a dotted line for comparison.

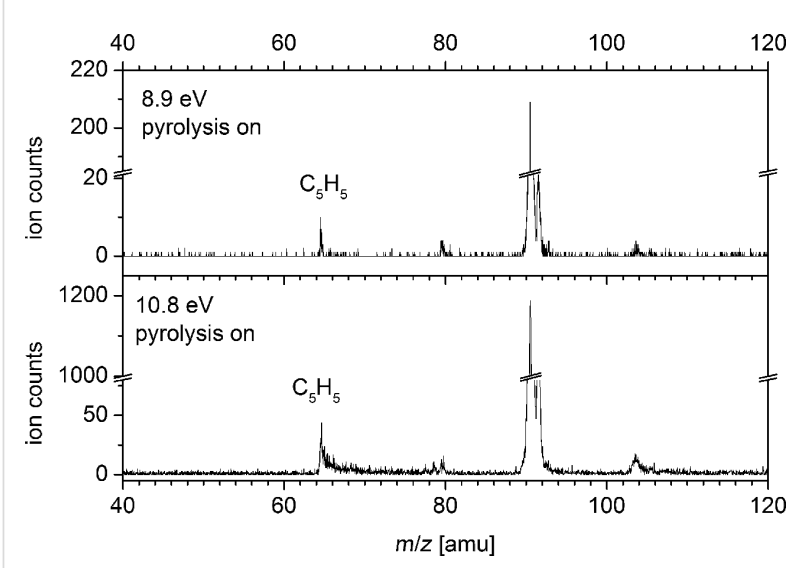

Figure 5: The shape of the $\mathrm{C}_{5} \mathrm{H}_{5}{ }^{+}$peak in the mass spectrum changes with photon energy. While the peak is symmetric at $8.9 \mathrm{eV}$, it shows a pronounced asymmetry at $10.8 \mathrm{eV}$, indicating the onset of dissociative photoionisation.

becomes asymmetric, as visible in the lower trace of Figure 5. Such an asymmetry indicates that the ion is a dissociation product of a metastable parent ion [40]. Formation of the cyclopentadienyl ion and acetylene upon dissociative photoionisation of tropyl can explain the rise in the mass channel above $10.55 \mathrm{eV}$ and the asymmetric peak shape. Thermochemical calculations reveal that the channel is accessible at $10.52 \mathrm{eV}$, utilizing the heat of formation at $0 \mathrm{~K}$ of $\mathrm{C}_{7} \mathrm{H}_{7}{ }^{+}\left(896 \mathrm{~kJ} \mathrm{~mol}^{-1}\right)$ [41], the $\Delta_{\mathrm{f}} H^{\mathrm{o}}$ of cyclopentadienyl radical $\left(276 \mathrm{~kJ} \mathrm{~mol}^{-1}\right)$ [42], its adiabatic IE $\left(8.428 \mathrm{eV}=813.18 \mathrm{~kJ} \mathrm{~mol}^{-1}\right)$ [39], and the $\Delta_{\mathrm{f}} H^{\mathrm{o}}$ of acetylene $\left(226.88 \mathrm{~kJ} \mathrm{~mol}^{-1}\right)$ [43]. This corresponds to around $4.3 \mathrm{eV}$ internal energy in the ion before it dissociates, being in good agreement with our experimentally observed onset value of $10.55 \mathrm{eV}$. As pyrolysis is not complete, one has to consider dissociative photoionisation of the precursor as a possible source of $\mathrm{C}_{5} \mathrm{H}_{5}{ }^{+}$. The signal in the $\mathrm{m} / \mathrm{z}=182$ mass channel, corresponding most likely to an isomer of bitropyl, is given as a dotted line in Figure 4. As can be seen, the signal is small throughout the studied energy range.

Between $7 \mathrm{eV}$ and $8.5 \mathrm{eV}$ the spectrum of tropyl has a Franck-Condon gap, and the transition to the first excited electronic state of the ion is visible at $9.65 \mathrm{eV}$. This peak has a shoulder at $9.85 \mathrm{eV}$ photon energy, i.e., $200 \mathrm{meV}$ higher, which may correspond to either vibrational excitation of the first electronic excited state or to the next electronic state. A noisy feature appears at $10.7 \mathrm{eV}$, and is followed by the highest intensity peak at $11.6 \mathrm{eV}$.

The most stable triplet state has been observed before at $9.63 \mathrm{eV}$ [16], and is calculated to lie $3.1 \mathrm{eV}$ above the ground state [9], i.e., at $9.33 \mathrm{eV}$ using the newly determined adiabatic ionisation energy of $6.23 \mathrm{eV}$. In the same work, Lee and Wright predict the next triplet state at $3.9 \mathrm{eV}$ excitation energy, i.e., at $10.13 \mathrm{eV}$ photon energy. We calculated the EOM-CCSD/ccpVTZ excitation energies for the triplet states, using QChem 4 [44] at the DFT-optimised ground state tropyl ion geometry, to be 3.82 and $4.00 \mathrm{eV}$, corresponding to 10.05 and $10.23 \mathrm{eV}$ photon energies. TD-DFT calculations yield 9.70, $10.05 \mathrm{eV}$ (B3LYP/6-311++G(d,p)); 9.86, 10.25 (M06-2X/6$311++\mathrm{G}(\mathrm{d}, \mathrm{p}))$ and 9.85, $10.03 \mathrm{eV}(\mathrm{BLYP} / 6-311 \mathrm{G}(\mathrm{d}, \mathrm{p}))$. Note that both triplet states are of E symmetry. The observed excitation energies are lower than the calculated values, which is due to possible Jahn-Teller distortions in the doubly degenerate triplet states, not considered in calculations of vertical excitation energies. The shoulder at $9.85 \mathrm{eV}$ may thus either be due to the second triplet state, or to a vibrational fundamental of the excited state. For example a $\mathrm{C}-\mathrm{C}$ stretching mode may be responsible for this peak, $1600 \mathrm{~cm}^{-1}$ further to the blue with respect to the first one.

The electronic spectroscopy of mass-selected tropyl cations has recently been investigated in Ne matrix [45]. A progression starting at $275.1 \mathrm{~nm}(4.51 \mathrm{eV})$ was assigned to the ${ }^{1} \mathrm{~A}_{2}$ " $\leftarrow \widetilde{X}^{+}{ }^{1} \mathrm{~A}_{1}$ ' transition of the tropyl cation, corresponding to $10.74 \mathrm{eV}$ in the photoelectron spectrum for the first excited singlet state of the ion. The shoulder at around $10.7 \mathrm{eV}$ in Figure 4 might be tentatively assigned to this state. However, previous calculations predicted the first singlet excited state at photon energies of 11.3 [9] and $11.74 \mathrm{eV}$ [45]. Our EOMCCSD result at 11.07 and TD-DFT results at 11.09, 11.24 and $10.95 \mathrm{eV}$ with the B3LYP, M06-2X and BLYP functionals, respectively, agree reasonably well with the experimental result of Nagy et al. [45]. 
Overall the theoretical predictions are less consistent in the range of the maximum TPE signal at $11.6 \mathrm{eV}$ (Figure 4). Lee and Wright reported a further triplet state at $12.4 \mathrm{eV}$, followed by a $1 \mathrm{eV}$ gap to the next electronic excited state. EOM-CCSD vertical-excitation-energy calculations predict that the next higher lying state is a triplet at $12.48 \mathrm{eV}$ followed by two more triplet states in ca. $100 \mathrm{meV}$ intervals as well as several singlet states around $12.8 \mathrm{eV}$. TD-DFT calculations, on the other hand, depend greatly on the functional used. B3LYP calculations agree best with the experiment, yielding both a singlet and a triplet state in the 11.8-11.9 eV photon energy range. BLYP yields almost a continuum of states at $11.38(\mathrm{~S}), 11.45(\mathrm{~T})$, $11.54(\mathrm{~S}), 11.60(\mathrm{~S})$ and $11.66(\mathrm{~S}) \mathrm{eV}$, whereas the next singlet state above $11.24 \mathrm{eV}$ is obtained at $12.05 \mathrm{eV}$ with the M06-2X functional. To summarize, wave-function methods, such as CIS, employed by Lee and Wright or EOM-CCSD predict a sparse electronic excitation spectrum with a gap at the experimentally observed main peak at $11.6 \mathrm{eV}$. Density functional results, on the other hand, are inconsistent in this energy range. Thus an unequivocal assignment of this band is difficult.

\section{Conclusion}

We studied the photoionisation of the tropyl radical, generated by pyrolysis of bitropyl, employing the iPEPICO technique. The first band in the mass-resolved threshold photoelectron spectrum at $6.23 \mathrm{eV}$ was assigned to the adiabatic ionisation energy. This value is in very good agreement with a previous extrapolation of Rydberg states. With the help of a Franck-Condon simulation two progressions were assigned. The first includes the vibration $v_{16}{ }^{+}$, an $e_{3}$, $\mathrm{C}-\mathrm{C}$ stretching mode with a spacing of $1530 \mathrm{~cm}^{-1}(0.19 \mathrm{eV})$, while the second progression is a combination of the $v_{2}{ }^{+} a_{1}$ ' ring-breathing mode and $v_{16}{ }^{+}$. The simulations also indicate activity in the $v_{17}{ }^{+} \mathrm{C}-\mathrm{H}$ in-plane bending mode of $e_{3}$ ' symmetry. Moreover the first triplet and (possibly) singlet excited states of the tropyl ion were observed at $9.65 \mathrm{eV}$ and $10.7 \mathrm{eV}$, respectively, in agreement with earlier work $[5,16]$. The second triplet state may also be visible at $9.85 \mathrm{eV}$. The most intense band appears at $11.6 \mathrm{eV}$. Computing this part of the spectrum proved challenging, with wave-function methods predicting a gap in this energy range, while DFT results depend greatly on the functional used. At around $10.55 \mathrm{eV}$ (4.3 eV internal energy) the tropyl ion starts to photoionize dissociatively to form the cyclopentadienyl ion. This value is in very good agreement with the appearance energy estimated from a thermochemical cycle.

\section{Experimental}

The experiments were carried out at the VUV beamline of the Swiss Light Source at the Paul Scherrer Institut (PSI) in Villigen, Switzerland. The beamline has been described in detail elsewhere $[46,47]$. The X04DB bending magnet provides synchrotron radiation, which is collimated and sent to a plane grating monochromator with a 600 grooves $/ \mathrm{mm}$ with a maximum resolving power of $10^{4}$. A mixture of $10 \% \mathrm{Kr}, 30 \%$ $\mathrm{Ar}$ and $60 \% \mathrm{Ne}$ at a pressure of 10 mbar was used to suppress radiation at higher harmonics in a differentially pumped gas filter. Below $7 \mathrm{eV}$ a $\mathrm{MgF}_{2}$ window was used instead of the gas mixture. A photon energy resolution of $5 \mathrm{meV}$ was achieved at $15.764 \mathrm{eV}$, measured at the $11 \mathrm{~s}$ resonance of argon.

The iPEPICO (imaging photoelectron photoion coincidence) technique was employed to study the photoionisation of tropyl 1. This technique allows the mass-selective detection of threshold photoelectrons by detecting them in coincidence with ions. The spectrometer is a combination of a Wiley-McLaren time-of-flight (TOF) mass spectrometer [48] and a velocity map imaging setup [49]. The latter is equipped with a position sensitive detector with a delay line anode (Roentdek DLD40). Only the central part of the electron image, corresponding to an electron energy resolution of around $5 \mathrm{meV}$, was taken for further analysis. The contribution of hot background electrons was subtracted following the method outlined by Sztáray and Baer [50].

A flange equipped with a molecular beam source and a SiC tube for flash pyrolysis [51] was mounted to the vacuum chamber. Bitropyl 3 was synthesized according to the literature [5], placed in an oven and heated to $90-105{ }^{\circ} \mathrm{C}$ to obtain a sufficient vapour pressure. The precursor was seeded in an argon flow of around 70 mbar and expanded through a $0.1 \mathrm{~mm}$ pinhole into the pyrolysis tube. The oven was mounted in line with the gas flow. An unskimmed jet was employed. The photon energy was scanned in steps of $5 \mathrm{meV}$ in the region of the ionisation threshold and $10-50 \mathrm{meV}$ in the higher energy regions. Data were averaged for 60 seconds per data point.

\section{Acknowledgements}

Financial support by the Deutsche Forschungsgemeinschaft (DFG) within the framework of the research training school GRK 1221 and through contract FI575/7-2 is gratefully acknowledged. This research project has been supported by the European Commission under the $7^{\text {th }}$ Framework Program, Research Infrastructures, grant agreement number 226716. The calculations were performed at the Leibniz-Rechenzentrum der Bayerischen Akademie der Wissenschaften (LRZ Munich). The iPEPICO experiments were carried out at the VUV beamline of the Swiss Light Source, Paul Scherrer Institut. We would like to thank Terry Miller and Gyorgy Tarczay for providing us with details of their computational results. The work was financially supported by the Swiss Federal Office for Energy (BFE Contract Number 101969/152433). 


\section{References}

1. Chatgilialoglu, C.; Studer, A., Eds. Encyclopedia of Radicals in Chemistry, Biology and Materials; John Wiley \& Sons: Chichester, 2012. doi:10.1002/9781119953678

2. Alcaraz, C.; Fischer, I.; Schröder, D. Radical Chemistry in the Gas Phase. In Encyclopedia of Radicals in Chemistry, Biology and Materials; Chatgilialoglu, C.; Studer, A., Eds.; John Wiley \& Sons: Chichester, 2012; Vol. 1, pp 477-502.

3. Thrush, B. A.; Zwolenik, J. J. Discuss. Faraday Soc. 1963, 35, 196-200. doi:10.1039/df9633500196

4. Elder, F. A.; Parr, A. C. J. Chem. Phys. 1969, 50, 1027-1028. doi:10.1063/1.1671081

5. Doering, W. V. E.; Knox, L. H. J. Am. Chem. Soc. 1957, 79, 352-356. doi:10.1021/ja01559a030

6. Fateley, W. G.; Lippincott, E. R. J. Chem. Phys. 1957, 26, 1471-1481. doi:10.1063/1.1743566

7. Sourisseau, C.; Hervieu, J. Spectrochim. Acta, Part A 1978, 34, 881-887. doi:10.1016/0584-8539(78)80007-5

8. Howard, J.; Graham, D. Spectrochim. Acta, Part A 1985, 41, 815-824. doi:10.1016/0584-8539(85)80027-1

9. Lee, E. P. F.; Wright, T. G. J. Phys. Chem. A 1998, 102, 4007-4013. doi:10.1021/jp980821e

10. Wood, D. E.; McConnell, H. M. J. Chem. Phys. 1962, 37, 1150-1151. doi:10.1063/1.1733231

11. Silverstone, H. J.; Wood, D. E.; McConnell, H. M. J. Chem. Phys. 1964, 41, 2311-2323. doi:10.1063/1.1726265

12. Smith, B. J.; Hall, N. E. Chem. Phys. Lett. 1997, 279, 165-171. doi:10.1016/S0009-2614(97)01016-6

13. Stakhursky, V. L.; Sioutis, I.; Tarczay, G.; Miller, T. A. J. Chem. Phys. 2008, 128, 084310. doi:10.1063/1.2829437

14. Sioutis, I.; Stakhursky, V. L.; Tarczay, G.; Miller, T. A. J. Chem. Phys. 2008, 128, 084311. doi:10.1063/1.2829471

15. Satink, R. G.; Meijer, G.; von Helden, G. J. Am. Chem. Soc. 2003, 125, 15714-15715. doi:10.1021/ja038329i

16. Koenig, T.; Chang, J. C. J. Am. Chem. Soc. 1978, 100, 2240-2242. doi:10.1021/ja00475a050

17. Pino, T.; Güthe, F.; Ding, H.; Maier, J. P. J. Phys. Chem. A 2002, 106, 10022-10026. doi:10.1021/jp020910y

18. Baer, T.; Guyon, P.-M. In High Resolution Laser Photoionisation and Photoelectron Studies; Powis, I.; Baer, T.; Ng, C. Y., Eds.; John Wiley \& Sons: New York, NY, 1995.

19. Baer, T. Int. J. Mass Spectrom. 2000, 200, 443-457. doi:10.1016/S1387-3806(00)00327-4

20. Baer, T.; Song, Y.; Liu, J.; Chen, W.; Ng, C. Y. Faraday Discuss. 2000, 115, 137-145. doi:10.1039/a909621c

21. Baer, T.; Sztáray, B.; Kercher, J. P.; Lago, A. F.; Bödi, A.; Skull, C.; Palathinkal, D. Phys. Chem. Chem. Phys. 2005, 7, 1507-1513. doi:10.1039/b502051d

22. Schüßler, T.; Deyerl, H.-J.; Dümmler, S.; Fischer, I.; Alcaraz, C.; Elhanine, M. J. Chem. Phys. 2003, 118, 9077-9080. doi:10.1063/1.1576387

23. Schüßler, T.; Roth, W.; Gerber, T.; Fischer, I.; Alcaraz, C. Phys. Chem. Chem. Phys. 2005, 7, 819-825. doi:10.1039/b414163f

24. Hemberger, P.; Steinbauer, M.; Schneider, M.; Fischer, I.; Johnson, M.; Bodi, A.; Gerber, T. J. Phys. Chem. A 2010, 114, 4698-4703. doi:10.1021/jp9068569

25. Hemberger, P.; Noller, B.; Steinbauer, M.; Fischer, I.; Alcaraz, C. Cunha de Miranda, B. K.; Garcia, G. A.; Soldi-Lose, H. J. Phys. Chem. A 2010, 114, 11269-11276. doi:10.1021/jp104019d
26. Steinbauer, M.; Hemberger, P.; Fischer, I.; Bodi, A. ChemPhysChem 2011, 12, 1795-1797. doi:10.1002/cphc.201000892

27. Xing, X.; Reed, B.; Lau, K.-C.; Ng, C. Y.; Zhang, X.; Ellison, G. B. J. Chem. Phys. 2007, 126, 171101. doi:10.1063/1.2737443

28. Gasser, M.; Schulenburg, A. M.; Dietiker, P. M.; Bach, A.; Merkt, F.; Chen, P. J. Chem. Phys. 2009, 131, 014304. doi:10.1063/1.3157185

29. Gao, H.; Xu, Y.; Yang, L.; Lam, C.-S.; Wang, H.; Zhou, J.; Ng, C. Y. J. Chem. Phys. 2011, 135, 224304. doi:10.1063/1.3664864

30. Taatjes, C. A.; Hansen, N.; Osborn, D. L.; Kohse-Höinghaus, K.; Cool, T. A.; Westmoreland, P. R. Phys. Chem. Chem. Phys. 2008, 10, 20-34. doi:10.1039/b713460f

31. Cunha de Miranda, B. K.; Alcaraz, C.; Elhanine, M.; Noller, B.; Hemberger, P.; Fischer, I.; Garcia, G.; Soldi-Lose, H.; Gans, B.; Viera Mendez, L. A.; Boyé-Péronne, S.; Douin, S.; Zabka, J.; Botschwina, P. J. Phys. Chem. A 2010, 114, 4818-4830. doi:10.1021/jp909422q

32. Johnson, R. D. J. Chem. Phys. 1991, 95, 7108-7113. doi:10.1063/1.461387

33. Eiden, G. C.; Lu, K.-T.; Badenhoop, J.; Weinhold, F.; Weisshaar, J. C. J. Chem. Phys. 1996, 104, 8886-8895. doi:10.1063/1.471624

34. Spangenberg, D.; Imhof, P.; Kleinermanns, K. Phys. Chem. Chem. Phys. 2003, 5, 2505-2514. doi:10.1039/b301228j

35. Gaussian 09, Revision B.01; Gaussian, Inc.: Wallingford, CT, 2010.

36. Houle, F. A.; Beauchamp, J. L. J. Am. Chem. Soc. 1978, 100, 3290-3294. doi:10.1021/ja00479a005

37. Roth, W. R.; Hunold, F.; Neumann, M.; Bauer, F. Liebigs Ann. 1996, 1679-1690. doi:10.1002/jlac.199619961029

38. DeFrees, D. J.; Mclver, R. T., Jr.; Hehre, W. J. J. Am. Chem. Soc. 1980, 102, 3334-3338. doi:10.1021/ja00530a005

39. Wörner, H. J.; Merkt, F. Angew. Chem., Int. Ed. 2005, 45, 293-296. doi:10.1002/anie.200503032

40. Sztáray, B.; Bodi, A.; Baer, T. J. Mass Spectrom. 2010, 45, 1233 doi:10.1002/jms.1813

41. Shin, S. K. Chem. Phys. Lett. 1997, 280, 260-265. doi:10.1016/S0009-2614(97)01145-7

42. Nunes, P. M.; Agapito, F.; Cabral, B. J. C.; dos Santos, R. M. B.; Simões, J. A. M. J. Phys. Chem. A 2006, 110, 5130-5134. doi:10.1021/jp060325n

43. Stull, D. R.; Prophet, H. JANAF Thermochemical tables, 2nd ed.; Midland: Michigan, 1971.

44. Shao, Y.; Molnar, L. F.; Jung, Y.; Kussmann, J.; Ochsenfeld, C.; Brown, S. T.; Gilbert, A. T. B.; Slipchenko, L. V.; Levchenko, S. V.; O'Neill, D. P.; DiStasio, R. A., Jr.; Lochan, R. C.; Wang, T.; Beran, G. J. O.; Besley, N. A.; Herbert, J. M.; Lin, C. Y.; Voorhis, T. V.; Chien, S. H.; Sodt, A.; Steele, R. P.; Rassolov, V. A.; Maslen, P. E.; Korambath, P. P.; Adamson, R. D.; Austin, B.; Baker, J.; Byrd, E. F. C. Dachsel, H.; Doerksen, R. J.; Dreuw, A.; Dunietz, B. D.; Dutoi, A. D.; Furlani, T. R.; Gwaltney, S. R.; Heyden, A.; Hirata, S.; Hsu, C.-P.; Kedziora, G.; Khalliulin, R. Z.; Klunzinger, P.; Lee, A. M.; Lee, M. S.; Liang, W.; Lotan, I.; Nair, N.; Peters, B.; Proynov, E. I.;

Pieniazek, P. A.; Rhee, Y. M.; Ritchie, J.; Rosta, E.; Sherrill, C. D.; Simmonett, A. C.; Subotnik, J. E.; Woodcock, H. L., III; Zhang, W.; Bell, A. T.; Chakraborty, A. K.; Chipman, D. M.; Keil, F. J.; Warshel, A.; Hehre, W. J.; Schaefer, H. F., III; Kong, J.; Krylov, A. I.; Gill, P. M. W.; Head-Gordon, M. Phys. Chem. Chem. Phys. 2006, 8, 3172-3191. doi:10.1039/b517914a

45. Nagy, A.; Fulara, J.; Garkusha, I.; Maier, J. P. Angew. Chem., Int. Ed. 2011, 50, 3022-3025. doi:10.1002/anie.201008036

46. Bodi, A.; Johnson, M.; Gerber, T.; Gengeliczki, Z.; Sztáray, B.; Baer, T. Rev. Sci. Instrum. 2009, 80, 034101. doi:10.1063/1.3082016 
47. Johnson, M.; Bodi, A.; Schulz, L.; Gerber, T.

Nucl. Instrum. Methods Phys. Res., Sect. A 2009, 610, 597-603.

doi:10.1016/j.nima.2009.08.069

48. Bodi, A.; Sztáray, B.; Baer, T.; Johnson, M.; Gerber, T.

Rev. Sci. Instrum. 2007, 78, 084102. doi:10.1063/1.2776012

49. Whitaker, B. J., Ed. Imaging in Molecular Dynamics; Cambridge University Press: Cambridge, 2003. doi:10.1017/CBO9780511535437

50. Sztáray, B.; Baer, T. Rev. Sci. Instrum. 2003, 74, 3763-3768.

doi:10.1063/1.1593788

51. Kohn, D. W.; Clauberg, H.; Chen, P. Rev. Sci. Instrum. 1992, 63, 4003-4005. doi:10.1063/1.1143254

\section{License and Terms}

This is an Open Access article under the terms of the Creative Commons Attribution License

(http://creativecommons.org/licenses/by/2.0), which permits unrestricted use, distribution, and reproduction in any medium, provided the original work is properly cited.

The license is subject to the Beilstein Journal of Organic Chemistry terms and conditions:

(http://www.beilstein-journals.org/bjoc)

The definitive version of this article is the electronic one which can be found at: doi:10.3762/bjoc. 9.77 